\title{
Effect of the acupoints ST-36 (Zusanli) and SP-6 (Sanyinjiao) on intestinal myoelectric activity of Wistar rats
}

\author{
A. Tabosal, \\ Y. Yamamura ${ }^{1}$, \\ E.R. Forno ${ }^{1}$ and \\ L.E.A.M. Mello ${ }^{2}$
}

\author{
${ }^{1}$ Divisão de Medicina Chinesa e Acupuntura, \\ Departamento de Ortopedia e Traumatologia, and \\ ${ }^{2}$ Departamento de Fisiologia, Universidade Federal de São Paulo, \\ São Paulo, SP, Brasil
}

\section{Correspondence \\ L.E.A.M. Mello \\ Departamento de Fisiologia \\ UNIFESP \\ Rua Botucatu, 862 \\ 04023-062 São Paulo, SP \\ Brasil \\ Fax: +55-11-5579-2033 \\ E-mail: lemello@ecb.epm.br}

Research supported by FAPESP (No. 99/06114-9). A. Tabosa was the recipient of a FAPESP fellowship.

Received August 30, 2001 Accepted April 3, 2002

\section{Abstract}

Despite its ancient use as a therapeutic tool to treat several ailments, acupuncture still faces the challenge of scrutiny by Western science both in terms of its efficacy and in terms of the characterization of its effects and mechanisms of actions underlying these effects. We investigated under well-controlled and carefully characterized conditions the influence of electrical stimulation of acupuncture points ST36 (Zusanli) and SP-6 (Sanyinjiao) on the myoelectric activity of the small intestine of 38 adult male Wistar rats. Electrical recordings obtained by means of four electrodes chronically implanted in the small intestine were used to assess the effects of acupuncture (electroacupuncture stimulation set at $2 \mathrm{~Hz}$, intermittent stimulation, $1 \mathrm{~V}$, for $30 \mathrm{~min}$ ). Immobilization of the animals was associated with a consistent decrease $(-8 \pm 7 \%)$ in the myoelectric activity of the small intestine as measured by means of the root mean square. Conversely, acupuncture was able to significantly increase (overshoot) this activity compared to baseline $(+44 \pm 7 \%)$. In contrast, immobilized animals subjected to sham acupuncture had only modest (nonsignificant) increases in myoelectric activity $(+9 \pm 6 \%)$. Using carefully controlled conditions we confirmed previous noncontrolled studies on the ability of acupuncture to alter intestinal motility. The characterization of the topographic and temporal profiles of the effects observed here represents a basis for future dissection of the physiological and pharmacological systems underlying these effects.

\section{Introduction}

The motor pattern of the small intestine can be classified into two main states, fasted and postprandial (1), which represent fundamentally different physiological states $(2,3)$. During fasting the motor activity of the small intestine shows a highly organized pattern and is characterized by a sequence of specif-

\section{Key words}

- Acupuncture

- Intestinal motility

- Rat

- Myoelectric activity

- Acupuncture point 
For acupuncture, a technique initially developed in China over 5,000 years ago, the "energetic harmony" of the digestive tube might be achieved through the stimulation of specific points such as ST-36 (Zusanli) and SP-6 (Sanyinjiao) $(10,11)$. Accordingly, acupuncture can be used to successfully treat various different human gastrointestinal conditions (12-14). Despite its wide clinical acceptance in Eastern societies, to date very little work has been done at the experimental level to test the efficacy of acupuncture stimulation under carefully controlled conditions and to address the features of such effects in areas other than analgesia $(12,15)$.

The relatively few investigations that have been devoted to the study of the effects of acupuncture on the digestive tract have either provided only a subjective assessment of the therapy or lacked adequate controls. $\mathrm{Hu}$ and colleagues (13) and Lin and colleagues (14) have both described the consequences of acupuncture for gastric motility in humans, but neither group has used a proper placebo group. In contrast, in a wellcontrolled study using anesthetized dogs, Qian and colleagues (16) have reported an excitatory effect of acupuncture with increased frequency and amplitude of the migrating motor complex of the stomach. Iwa and Sakita (17) reported similar excitatory effects for the peristaltic motility of the small intestine in mice. The latter study, however, has used a rather crude method for evaluating intestinal motility (charcoal transit), did not report whether the animals were anesthetized or restrained for needle insertion, and did not provide much additional relevant information regarding the experimental protocol.

Whereas the above mentioned studies represent an initial step towards the scientific investigation of the effects of acupuncture on the gastrointestinal system, they certainly represent a weak background to substantiate its clinical use. A study in the Journal of the American Medical Association has indicated that $42 \%$ of Americans used alternative medical therapies in 1997 (18). Acupuncture certainly is an important component of such percentage. The current study aims to provide a more detailed and adequate evaluation of acupuncture with objective measurements under carefully controlled conditions.

\section{Material and Methods}

Adult male Wistar rats from the Central Vivarium (CEDEME) of our University, weighing 250-290 $\mathrm{g}$ at the beginning of the experiment were used. Animals were kept in individual cages in a controlled environment with constant temperature $\left(23^{\circ} \mathrm{C}\right), 12$-h light/ dark cycles with lights on at 7:00 am and with free access to rat chow pellets (Purina, Nuvilab Ltda., Curitiba, PR, Brazil) and water. All experiments were submitted to and approved by the Animal Care and Use Committee of the University.

After a 12-h fast (with free access to water), animals were anesthetized with thionembutal, $50 \mathrm{mg} / \mathrm{kg}$, ip. The surgical procedure used has been described (19-21). The skull was exposed for the fixation of two small anchor screws which were attached to an electrical plug with dental acrylate. Nine electrically isolated copper wires connected to the electric plug were then inserted into the subcutaneous tissue of the neck and tunneled to the abdomen. The abdominal wall was opened and four bipolar copper electrodes with silver-chloride plating at their recording end were implanted in the small intestine. The first of these four electrodes was implanted $5 \mathrm{~cm}$ distal to the suspensory muscle of the duodenum (ligament of Treitz) (22) and the remaining three electrodes at subsequent distal portions spaced $7 \mathrm{~cm}$ apart. The remaining ninth wire (stainless steel) was used as ground and implanted in the subcutaneous fold of the right lateral abdominal wall.

After an average postoperative period of 
5 days, which has been reported to be enough to allow a recovery of the regular myoelectric pattern $(19,23,24)$, animals were randomly allocated to one of three groups: group I, electroacupuncture $(\mathrm{N}=13)$; group II, sham electroacupuncture $(\mathrm{N}=14)$, and group III, immobilized $(\mathrm{N}=11)$. In each group each animal served as its own control. A baseline recording of animals in the interdigestive state (fasting initiated $12 \mathrm{~h}$ before) was obtained immediately before each experimental procedure for a total of $90 \mathrm{~min}$. For this recording, animals were simply removed from their home cages and connected via the head plugs to a 16-channel electrical recording equipment (CAD 12/36, Lynx Tecnologia Eletrônica Ltda., São Paulo, SP, Brazil). Electric signals acquired at a sample frequency of $100 \mathrm{~Hz}$, with a high-pass cutoff filter set at $40 \mathrm{~Hz}$, were then processed and stored in a standard computer (PC, Pentium, $100 \mathrm{MHz}, 8 \mathrm{MB}$ RAM). During baseline recordings animals were unrestricted and remained in their individual home cages, which were placed inside a Faraday cage (50 $x 50 \times 50 \mathrm{~cm})$. Data analysis was performed with the Sisdin software (Lynx). For signal processing, recording sessions of the electromyographic signals of $90 \mathrm{~min}$ each were analyzed for every animal and the root mean square (RMS) was calculated as described elsewhere (25). RMS represents the intensity of a signal within a fixed interval. It is defined as the square root of the mean sum of spike bursts per minute squared (here over $90 \mathrm{~min}$ ) or by the equation:

$$
\mathrm{RMS}=\sqrt{\frac{1}{\mathrm{~T}}} \sum_{\mathrm{t}=1}^{\mathrm{t}=\mathrm{T}} f(\mathrm{t})^{2}
$$

where $\mathrm{T}$ is the size of the block of data (90 $\min$ ) and $f(\mathrm{t})=$ value (in spike bursts/min) of the filtered waveform at $t$ minus the mean of the entire block of data. RMS is an effective value representing the intensity of a signal within a fixed interval, regardless of the direction in which the signal is recorded.
Some of the recordings (9\%) made with specific leads were discarded due to technical problems such as loss of contact, poor baseline with excessive noise, and unsuitable signal to noise ratio. Therefore, the number of available recordings for each group is not necessarily the number of animals in that group times four (the number of electrode leads implanted in each animal).

After recording baseline activity, animals were divided into one of the above three groups. Group I animals (13 animals subjected to electroacupuncture, corresponding to a total of 48 useful recordings) were then restrained for $30 \mathrm{~min}$, during which time electroacupuncture $(2 \mathrm{~Hz}$, with an electrical current of faradic, bipolar and asymmetrical wave, at approximately $1-\mathrm{V}$ intensity; KWD 808-II electrostimulator, China) was applied through a stainless steel $0.25 \mathrm{~mm} / 2 \mathrm{~cm}$ needle with a copper handle bilaterally inserted into the ST-36 (Zusanli) and SP-6 (Sanyinjiao) points. The choice of these acupoints (points of acupuncture) was based on their reported abilities to stimulate intestinal activity (11, 12). The localization of such points was based both on rat anatomical references (26) and on a point localizer (basically an impedance meter; Plexus T20, Lautz, Rio Claro, SP, Brazil). In the rat, ST-36 (Zusanli) is located approximately $1 \mathrm{~mm}$ lateral to the tibial tuberosity, whereas SP-6 (Sanyinjiao) is located in the posterior border of the tibia, $0.5 \mathrm{~mm}$ above the medial malleolus, both of which are easily located by manual inspection (see Figure 1). The needle was inserted into the ST-36 point at a depth of approximately $0.5 \mathrm{~mm}$, and just above the skin at the SP-6 point (as there is no great muscular mass under this area). A number of reports on different animal species have clearly demonstrated that the location of acupuncture points follows a similar distribution in different mammals (for a review, see 27). The use of impedometers allows the clear definition of points and nearby non-points (2730). Point localizers are often used in both 
Figure 1. Photograph depicting the precise location of needle insertion in a group I animal (electroacupuncture). The needle (see insertion marked with a black dot) at the most distal part of the hind limb corresponds to point SP-6 (Sanyinjiao) and the more proximal needle (see black dot) corresponds to ST-36 (Zusanli). White dots correspond to stimulation points used for the sham group (group II).

human and veterinary acupuncture to find the appropriate acupuncture points. Anatomical studies have shown that acupuncture points have a specific histological pattern (e.g., more mast cells, higher density of sensory receptors and capillary vessels) which correlates with a lower electrical resistance of the underlying skin (31-33). After application of electroacupuncture the animal was placed back in its home cage, allowed a 10min resting period, and then subjected to a subsequent 90 -min period of electrical recording of myoelectric activity. Data analysis for all groups was based on a comparison of the data obtained before and after the procedure.

For group II animals (14 animals subjected to sham acupuncture, corresponding to a total of 52 useful recordings) all procedures were similar to those used for group I

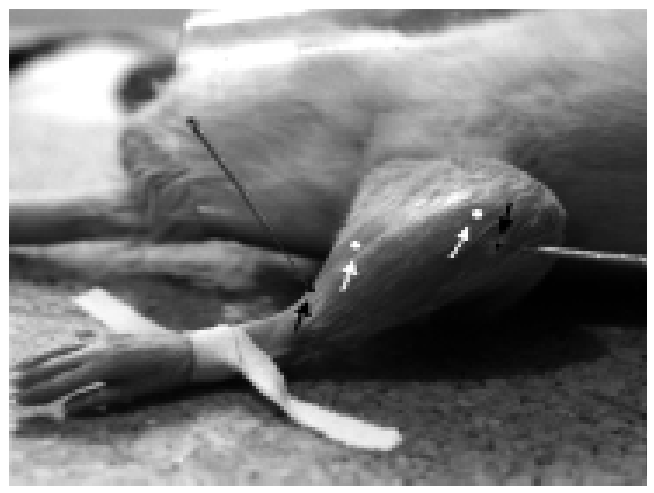

Table 1. Change in root mean square of the myoelectric activity recorded in the proximal $1 / 3$ of the small intestine at four different locations for each of the three experimental groups (electroacupuncture, sham electroacupuncture and immobilized) compared to the baseline condition (control recording).

Electroacupuncture $(\mathrm{N}=48)$ Sham electroacupuncture $(N=52)$ Immobilized $(\mathrm{N}=40)$ $44 \pm 7 *$ $9 \pm 6$ $-8 \pm 7$

Data are reported as means \pm SEM of the percent variation over baseline recording for each animal. $\mathrm{P}=0.0001$ (ANOVA followed by Fisher PLSD post hoc test).

$* \mathrm{P}<0.05$ compared to immobilized or to shamstimulated animals. animals except that electrostimulation was applied to neighboring skin areas (less than a 0.5 -cm distance from the acupoints) for which impedance analysis did not reveal a lower skin resistance compared to adjacent areas. Location of the stimulation points used for group I and II animals is illustrated in Figure 1. Finally, for group III animals (11 animals subjected to immobilization, corresponding to a total of 40 useful recordings) after the baseline recording animals were restrained for $30 \mathrm{~min}$, returned to their home cages, allowed a 10-min recovery period, and then subjected to a 90-min recording session. Electrical activity for each condition was normalized for its specific control (baseline recording). Data are reported as mean variations from control \pm SD. Positive values indicate increases in the parameters of myoelectrical activity, whereas negative values indicate decreases in these activities.

Statistical differences were first determined by one-way analysis of variance (ANOVA), followed by the Fisher PLSD test for post hoc comparisons. Comparisons between different times for a given group were performed by repeated measures ANOVA followed by the Fisher PLSD test for post hoc comparisons. The level of significance was set at $\mathrm{P}<0.05$ in all tests.

\section{Results}

Immobilization of the animals was associated with a consistent decrease in the myoelectric activity of the small intestine (Table 1). Conversely, acupuncture applied to points ST-36 (Zusanli) and SP-6 (Sanyinjiao) in immobilized animals not only normalized this diminished activity but also increased it (overshoot) compared to baseline (see Figure 2). In contrast, immobilized animals subjected to sham acupuncture had only modest (nonsignificant) increases in myoelectric activity. Thus, acupuncture induced a significant increase in the myoelectric activity of the small intestine compared to either 
sham acupuncture or immobilization alone (see Table 1).

Analysis of the results for the three groups tested for each of the four recording leads yielded a more detailed view of the effects (see Table 2). Results from leads T1 and T2 (more proximal recording sites) consistently indicated a statistically significant excitatory action of acupuncture compared to immobilization alone or to sham acupuncture. In contrast, recordings from leads $\mathrm{T} 3$ and $\mathrm{T} 4$ did not permit a significant distinction between animals subjected to acupuncture and animals subjected to sham acupuncture. Yet, even at the level of the T4 lead the increase in myoelectric activity induced by acupuncture was significantly different from the recordings obtained from immobilized animals.

Of the 13 animals that constituted group I, 4 (31\%) showed increases in myoelectric activity for all four recording leads. For the remaining 9 animals, the observed changes in myoelectric activity were either not uniform across the different leads (e.g., increase in $\mathrm{T} 1$ and $\mathrm{T} 2$ but not in $\mathrm{T} 3$ and $\mathrm{T} 4$ ) or were very modest changes of less than $15 \%$ of baseline. Of the 48 recordings obtained for group I animals none showed a decrease of myoelectric activity greater than $13 \%$ of baseline. The largest increase in myoelectric activity seen for group I animals was $188 \%$ over baseline (lead T2 of rat Amft11). For approximately one third of group I animals the increased activity was not uniform across the different leads. Intragroup variability for recordings from leads $\mathrm{T} 1$ and $\mathrm{T} 3$ of group I animals was rather small as compared to the variability seen at leads T2 and T4. Despite this variability, the excitatory effects of electroacupuncture were more intense at proximal (T1 and $\mathrm{T} 2$ ) than at distal recording sites (T3 and T4).

Analysis of myoelectric activity with time clearly showed the effects of acupuncture. A comprehensive view of the myoelectric activity of group I animals over time is provided in Figure 3. By separately analyzing the results shown in Figure 1 for each individual animal over time, it can be seen that, as early as during the first minute of the recording period (i.e., approximately $30 \mathrm{~min}$ after the onset of acupuncture stimulation and right after its termination) the myoelectric activity of 4 of the 13 group I animals (31\%) was increased by more than $50 \%$ over baseline. Similarly, consideration of the mean myoelectric activity of the initial $10 \mathrm{~min}$ of recording indicated that for 8 of those animals $(62 \%)$ there was an increase in activity greater than $20 \%$. Despite variations from animal to animal and over time, in group I animals as a whole acupuncture induced an increase of over $20 \%$ in myoelectric activity when the 10 -min period from the 31 st to the 40th min (therefore the middle part of the recording session) or from the 81 st to the 90 th min (the last 10-min period of the recording session) was considered in 53\% (7/13) and 67\% (6/9) of the animals, respectively. For some indi-

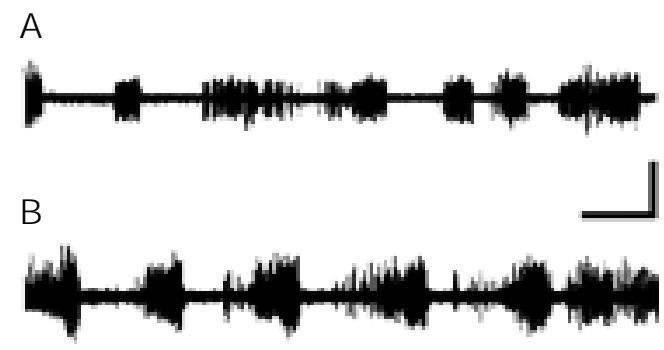

Figure 2. Electromyographic recordings from a group I animal (electroacupuncture) during baseline control recording prior to stimulation (A) and $10 \mathrm{~min}$ after electroacupuncture stimulation (B). Note the increase in the number and amplitude of the myoelectric motor complex in B compared to A. Calibration bars: $10 \mathrm{~min}$ and $100 \mathrm{mV}$.

Table 2. Change in root mean square of the myoelectric activity recorded in the proximal $1 / 3$ of the small intestine at four different locations for each of the three experimental groups (electroacupuncture, sham electroacupuncture and immobilized) compared to the baseline condition (control recording).

\begin{tabular}{lcccc}
\hline & \multicolumn{5}{c}{ Terminals } \\
\cline { 2 - 5 } & T1 & T2 & T3 & T4 \\
\hline Electroacupuncture $(\mathrm{N}=13)$ & $37 \pm 10^{*+}$ & $56 \pm 17^{*+}$ & $32 \pm 12$ & $49 \pm 18^{*}$ \\
Sham electroacupuncture $(\mathrm{N}=14)$ & $-4 \pm 14$ & $7 \pm 16$ & $19 \pm 9$ & $12 \pm 12$ \\
Immobilized $(\mathrm{N}=11)$ & $-8 \pm 12$ & $-8 \pm 16$ & $-11 \pm 16$ & $-5 \pm 10$ \\
\hline
\end{tabular}

Data are reported as means \pm SEM of the percent variation over baseline recording for each animal and terminal.

$P=0.0001$ (ANOVA followed by Fisher PLSD post hoc test).

$* \mathrm{P}<0.05$ compared to immobilized animals.

$+P \leq 0.05$ compared to sham-stimulated animals. 
vidual animals, however, the increase in myoelectric activity in these three time windows was even greater, as can be seen in Table 3.

For group II animals, variations were much more pronounced than those reported

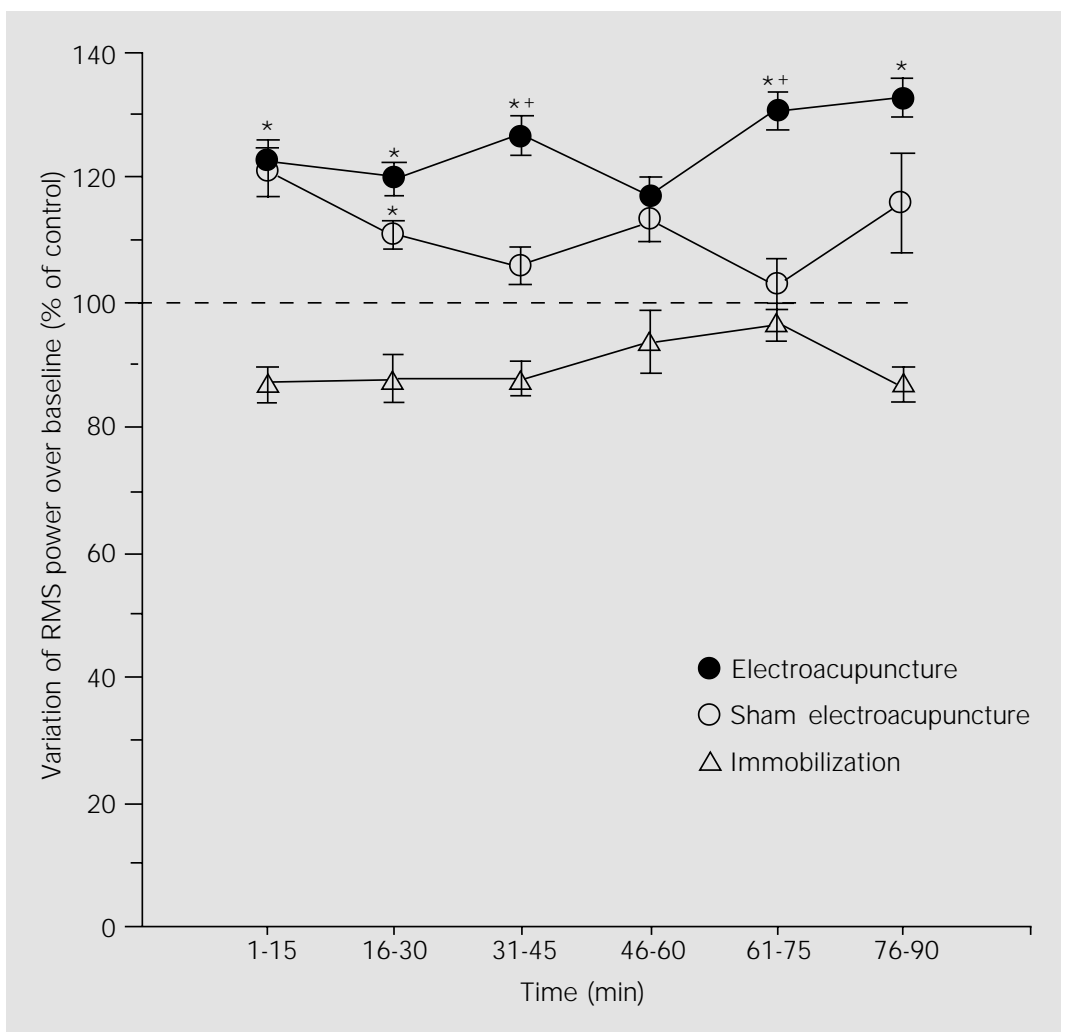

Figure 3. Variation of root mean square (RMS) power over baseline recordings of the intestinal myoelectric activity as measured over time in 6 consecutive time bins (each bin of 15-min duration). Electroacupuncture ( $\mathrm{N}=13$, filled circles), animals subjected to electroacupuncture at points ST-36 (Zusanli) and SP-6 (Sanyinjiao); sham electroacupuncture ( $\mathrm{N}=$ 14, open circles), animals with needle inserted and stimulated at non-acupuncture points; immobilized ( $\mathrm{N}=11$, triangles), animals subjected only to immobilization.

$\mathrm{P}=0.0001$ (repeated measures ANOVA).

$* \mathrm{P}=0.0001$ compared to immobilized animals (Scheffé F-post hoc test).

$+P=0.0001$ compared to sham animals (Scheffé F-post hoc test).

Table 3. Number of animals subjected to acupuncture stimulation (group I) with a greater than $20 \%$ increase in myoelectric activity over baseline, as measured by root mean square or a greater than $40 \%$ increase in myoelectric activity over baseline during the initial $10 \mathrm{~min}$ of recording (1-10) or in the middle portion of the recording session (31-40) or at the end of the recording session (81-90).

\begin{tabular}{llll}
\hline Increase & $1-10 \mathrm{~min}$ & $31-40 \mathrm{~min}$ & $81-90 \mathrm{~min}$ \\
\hline Greater than 20\% & $62(8 / 13)$ & $54(7 / 13)$ & $67(6 / 9)$ \\
Greater than 40\% & $23(3 / 13)$ & $54(7 / 13)$ & $44(4 / 9)$
\end{tabular}

above. Consistent increases in myoelectric activity across all recording leads were not seen for any given animal. Of the 14 animals that constituted this group, 6 (43\%) had increases in myoelectric activity at some leads coexisting with decreases at other recording leads. However, in 7 other animals dramatically decreased activity (a reduction of as much as $64 \%$ from baseline, lead $\mathrm{T} 2$ of rat AT008) recorded at some leads coexisted with either stable or increased myoelectric activity at others. Finally, for the remaining animal in group II, activity was close to baseline and quite uniform across all four leads. The largest increase in myoelectric activity seen for group II animals was of $148 \%$ over baseline (lead T2 of rat Amft10). Analysis of the myoelectric activity of group II animals at the individual level over time also did not yield any clear-cut picture. As can be seen in Figure 3, as a whole, animals subjected to sham acupuncture seemed to show a tendency to a slight decrease in myoelectric activity over time.

Animals subjected to immobilization only (group III animals) had the greatest decreases in myoelectric activity over baseline of all groups (up to a $86 \%$ reduction from baseline for lead $\mathrm{T} 3$ of rat Amft12). Variability across different leads, however, was similar to that seen for group II animals, with most of the rats having either combined increased/decreased (36\% of the animals) or combined stable/decreased (27\%) myoelectric activity compared to baseline. The largest increase in myoelectric activity seen for group III animals was $76 \%$ over baseline (lead T2 of rat Amft2). As shown in Figure 3, as a group, animals subjected to immobilization seem to have a tendency (not statistically significant) to recover to baseline levels (corresponding to 1 on the $y$ axis).

For animals subjected to sham acupuncture (group II animals) we observed a tendency, which did not achieve statistical significance, to increased myoelectric activity compared to restrained animals. Yet, despite 
this modest increase in myoelectric activity seen for group II animals, the mean activity was still significantly lower than that observed in group I animals $(P=0.01)$. Similarly to group III animals (immobilization), the results for group II animals were rather uniform across the four different leads. Analysis of the recordings as a whole revealed clear differences between the three groups $(P=0.0001)$, which were still significant for electrodes T1, T2 and T4, but not for electrode T3 $(\mathrm{P}=0.06)$, when analyzed separately.

\section{Discussion}

The current data confirm and expand the conclusions derived from previous work on this area (12-17). Similar results were obtained by Qian and colleagues (16) who demonstrated in dogs a significant increase in both the frequency and the amplitude of the slow waves of the migrating motor complex at the level of the stomach after the application of acupuncture at points ST-36 (Zusanli) and CS-6 (Neiguan). Also, Iwa and Sakita (17) have reported excitatory effects on small intestine motility of electroacupuncture applied to mice, although they did not describe the specific acupoints used. In contrast, work by $\mathrm{Hu}$ and colleagues (13) and Lin and colleagues (14) has demonstrated an inhibitory effect induced by acupuncture on tachyarrhythmia, nausea and dizziness in human subjects. These seemingly opposite results obtained with acupuncture in fact might reflect its balancing nature rather than an exclusive excitatory or inhibitory effect it might have. Accordingly, we propose that the excitatory effect reported here and elsewhere (see Refs. 16 and 17) might have to do with the depressed baseline of myoelectric activity of the small intestine that these animals had (or should have had) by being anesthetized or restrained. Even though the above mentioned work on laboratory animals does not report whether the animals were anesthetized or restrained it is clear that, in contrast to humans, it might be virtually impossible to keep an acupuncture needle inserted into a given site in a nonanesthetized unrestrained laboratory animal. Therefore, when applied over a depressed baseline activity, such as that seen in the current study, acupuncture yielded an excitatory, reactive effect. Conversely, when applied over a background of increased excitability, such as that present in human studies, it yielded an inhibitory and once again reactive effect. A recent demonstration of this overall compensatory effect of acupuncture has been made by Yao (34) based on the lowering of blood pressure observed when acupuncture was applied to hypertensive rats and the lack of any effect when it was applied to normotensive rats. Such a harmonizing effect might be present in a few situations even in Western medicine. One of such examples is the use of lithium salts as a therapeutic means both as a treatment of mania or depression (35).

Our results with the sham animals suggest that stimulation per se, regardless of whether applied to an acupoint or to a nearby non-acupoint, might induce physiologic effects. Yet, it is clear from the current results and from the extensive literature on acupuncture making use of sham controls, that as a rule the results obtained from stimulation of acupoints are greater and significantly different from those obtained from stimulation of non-acupoints. The difference between acupoints and non-acupoints might be that the skin and body areas associated with acupoints have been characterized as having a higher concentration of capillaries, sensory receptors and mast cells $(31,33)$. The difference between acupoints and nonacupoints would therefore be of a quantitative rather than a qualitative nature.

According to our data, the most intense effects of acupuncture on the myoelectric activity of the small intestine were found at the recording sites more proximal to the 
sponsory muscle of the duodenum (T1 and $\mathrm{T} 2)$. It has been shown that in the more proximal portions of the small intestine there may be a spontaneous oscillator, as opposed to a forced oscillator, due to its capacity to keep cycling even in the absence of external stimulation $(2,24,36)$. Despite its ability to keep cycling myoelectric activity in the absence of an extrinsic neural input (2), it has been shown that its activity might be modulated by such external influences (36). In fact, extrinsic neural or pharmacological influence is always stronger on this oscillator, i.e., at a proximal as compared to a more distal level $(37,38)$. The stronger effects of acupuncture on the myoelectric activity of the small intestine observed in the current experiments for the more proximal electrodes are therefore in agreement with the above findings.

The currently reported temporal profile of the effect of acupuncture on intestinal myoelectric activity constitutes an important additional characterization of such effect.
As shown in the Results section, the effects of acupuncture were already clear right at the first minute of recording, when individual animals were analyzed, and gradually increased with time over the subsequent 89 min of recording. The latency and duration of the observed effects of acupuncture are compatible with those reported for the effects of different pharmacological procedures on intestinal motility $(1,39,40)$. In addition, the results shown in Figure 3 clearly indicate that, whereas animals in the sham group differed from controls (animals subjected to immobilization) only at initial time points (from 1 to 15 and from 16 to $30 \mathrm{~min}$ ), animals in the acupuncture group significantly differed from controls at almost all time points. In this sense, analysis of the temporal profile of the effect of acupuncture might be of help in further dissociating its effect due to stimulation of a specific set of points (acupoints) from those only associated with the needling of nonspecific skin areas (nonacupoints).

\section{References}

1. Lördal $M$, Bränström $R \&$ Hellström $M$ (1998). Mediation of irregular spiking activity by multiple neurokinin-receptors in the small intestine of the rat. British J ournal of Pharmacology, 123: 63-70.

2. Sama SK (1985). Cyclic motor activity migrating motor complex. Gastroenterology, 89: 894-913.

3. Quigley EMM (1996). Gastric and small intestinal motility in health and disease. Gastroenterology Clinics of North America, 25: 113-145.

4. Ruckebusch M \& Fioramonti J (1975). Electrical spiking activity and propulsion in small intestine in fed and fasted rats. Gastroenterology, 68: 1500-1508.

5. Eagon J C \& Soper NJ (1993). Gastrointestinal pacing. Surgical Clinics of North America, 73: 1161-1172.

6. Stam R, Croiset G, Akkermans IMA \& Wiegant VM (1995). Effects of novelty and conditioned fear on small intestinal and colonic motility and behavior in the rat. Physiology and Behavior, 58: 803-809.
7. Chang EB, Sitrin MD \& Black DD (1996). Gastrointestinal, Hepatobiliary, and Nutritional Physiology. Lippincott-Raven, Philadelphia, PA, USA, 27-54.

8. Davenport HW (1978). Fisiologia do Trato Digestivo. 3rd edn. Guanabara Koogan, Rio de J aneiro, RJ , Brazil.

9. Wong M (1995). Ling-Shu-base de L'acupuncture Traditionnelle Chinoise. Masson Éditeur, Paris, France, 304-309.

10. Yamamura Y (1995). Acupuntura Tradicional - A Arte de Inserir. 2nd edn. Roca, São Paulo, SP, Brazil, 109-128.

11. Nghi NV (1981). Patogenia y Patologia Energeticas en Medicina China. Volume II, IAM, Madrid, Spain, 66-81.

12. Li Y, Tougas G, Chiverton SG \& Hunt RH (1992). The effect of acupuncture on gastrointestinal function and disorders. American J ournal of Gastroenterology, 87: 1372-1381.

13. Hu S, Stern RM \& Koch KL (1992). Electrical acustimulation relieves vectioninduced motion sickness. Gastroenterol- ogy, 102: 1854-1858

14. Lin X, Liang J, Ren J , Mu F, Zhang M \& Chen J D (1997). Electrical stimulation of acupuncture points enhances gastric myoelectrical activity in humans. American J ournal of Gastroenterology, 92: 1527-1530.

15. Litscher G, Wang L, Yang NH \& Schwarz G (1999). Computer-controlled acupuncture. Quantification and separation of specific effects. Neurological Research, 21: 530-534.

16. Qian L, Peters LJ \& Chen JD (1999). Effects of electroacupuncture on gastric migrating myoelectrical complex in dog. Digestive Diseases and Sciences, 44: 5662.

17. Iwa M \& Sakita M (1994). Effects of acupuncture and moxibustion on intestinal motility in mice. American J oumal of Chinese Medicine, 22: 119-125.

18. Astin J A (1988). Why patients use alternative medicine - Results of a national study. J ournal of the American Medical Associa- 
tion, 279: 1548-1553.

19. Fioramonti J \& Bueno I (1980). Gastrointestinal myoelectric activity disturbances in gastric ulcer disease in rats and dogs. Digestive Diseases and Sciences, 25: 575580.

20. Al-Saffar A (1984). Analysis of intestinal motility in fasted rats, with special reference to neurotensin. Scandinavian J ournal of Gastroenterology, 19: 422-428.

21. Wood J D (1981). Intrinsic neural control of intestinal motility. Annual Review of Physiology, 43: 33-51.

22. Piñeiro-Carrero $\mathbf{V M}$, Clench $M H$, Davis $\mathrm{RH}$, Andres J M, Franzini DA \& Mathias J R (1991). Intestinal motility changes in rats after enteric serotonergic neuron destruction. American J ournal of Physiology, 260 (Part 1): G232-G239.

23. Wittmann $T$, Crenner $F$, Angel $L$, Hanusz L, Ringwald C \& Grenier J F (1990). Longduration stress. Immediate and late effects on small and large intestine motility in rat. Digestive Diseases and Sciences, 35: 495-500.

24. Furness J B \& Costa M (1987). The Enteric Nervous System. Churchill Livingstone, Edinburgh, Scotland.

25. Zenilman ME, Parodi JE \& Becker J M (1992). Preservation and propagation of cyclic myoelectric activity after feeding in rat small intestine. American J oumal of Physiology, 263: G248-G253.

26. Romita W, Suk A \& Henry J L (1997).
Parametric studies on electroacupuncture-like stimulation in a rat model: effects of intensity, frequency, and duration of stimulation on evoked antinociception. Brain Research Bulletin, 42: 289-296.

27. Zhong-Xiang $Z$ (1981). Research advances in the electrical specificity of meridians and acupuncture points. American J ournal of Acupuncture, 9: 203-216.

28. Bergsmann O \& Woolley-Hart A (1973). Differences in electrical skin conductivity between acupuncture points and adjacent skin areas. American J ournal of Acupuncture, 1: 27-32.

29. Hu JH (1975). The neural basis of meridians and acupuncture points. American J ournal of Acupuncture, 3: 129-132.

30. Reichmanis $M$, Marino AA \& Becker RO (1979). Laplace plane analysis of impedance on the meridian. American J ournal of Chinese Medicine, 7: 188-193.

31. Zonglian H (1986). Study of receptors of sensation at somatic acupuncture points. In: Xiangtong Z (Editor), Research on Acupuncture, Moxibustion, and Acupuncture Anesthesia. Science Press Beijing, Hong Kong, China, 428-436.

32. Kendall DE (1989). Part I: A scientific model for acupuncture. American J ournal of Acupuncture, 17: 251-268.

33. Zhu Z \& Xu R (1990). Morphometric observation on the mast cells under the acupuncture meridian lines. Zhen $\mathrm{Ci}$ Yan $\mathrm{J}$ iu, 15: 157-158.
34. Yao T (1993). Acupuncture and somatic nerve stimulation: mechanism underlying effects on cardiovascular and renal activities. Scandinavian J ournal of Rehabilitation Medicine, 29 (Suppl): 7-18.

35. Malenka RC, Hamblin MW \& Barchas J D (1989). Biochemical hypotheses of affective disorders in anxiety. In: Siegel G, Agranoff B, Albers RW \& Molinoff P (Editors), Basic Neurochemistry. 4th edn. Raven Press, New York, NY, USA, 877891.

36. Zenilman ME (1993). Origin and control of gastrointestinal motility. Surgical Clinics of North America, 73: 1081-1099.

37. Nowak TV \& Harrington B (1985). Effect of cholinergic agonists on muscle from rodent proximal and distal small intestine. Gastroenterology, 88: 1118-1125.

38. Otterson MF \& Sarr MG (1993). Normal physiology of small intestine. Surgical Clinics of North America, 73: 1173-1192.

39. Fargeas MJ, Fioramonti J \& Bueno L (1993). Central action of interleukin $1 ß$ on intestinal motility in rats: mediation by two mechanisms. Gastroenterology, 104: 377 383.

40. Izzo AA, Mascolo N, Pinto L, Capasso R \& Capasso $F$ (1999). The role of cannabinoid receptors in intestinal motility, defaecation and diarrhoea in rats. European J ournal of Pharmacology, 384: 37-42. 